\title{
Estabilidade físico-química, microbiológica e sensorial de carne ovina embalada a vácuo estocada sob refrigeração
}

\author{
Physical and chemical stability microbiological and sensory analysis of vacuum packed sheep meat \\ stored under refrigeration
}

\author{
Rafaella de Paula Paseto Fernandes ${ }^{\mathrm{I}}$ Maria Teresa de Alvarenga Freire ${ }^{\mathrm{II}}$ Carlos Cirelli Guerra ${ }^{\mathrm{II}}$ \\ Celso da Costa Carrer ${ }^{\mathrm{III}}$ Júlio Cesar de Carvalho Balieiro ${ }^{\mathrm{IV}}$ Marco Antonio Trindade ${ }^{\mathrm{II}}$
}

\section{RESUMO}

A carne ovina é comercializada atualmente em sua quase totalidade na forma congelada, porém com a tendência atual de maior procura por produtos de conveniência, vislumbra-se a necessidade de o mercado começar a ofertar esta carne na forma de cortes refrigerados. Este trabalho teve como objetivo avaliar a estabilidade $e$ segurança de lombo de cordeiro embalado a vácuo quando estocado sob refrigeração. A estabilidade foi avaliada por meio de análises físicas e químicas (oxidação lipídica, cor objetiva, valor de $\mathrm{pH}$, composição centesimal, perda de água por cocção e textura instrumental), microbiológicas (contagem total de psicrotróficos anaeróbios, coliformes termotolerantes a $45^{\circ} \mathrm{C}$, estafilococos coagulase positiva e presença de Salmonella) e sensorial (cor, aparência geral e aroma). A carne ovina apresentou-se estável durante o período de 28 dias com relação à maioria dos índices físicos e químicos avaliados e dentro dos padrões estabelecidos pela legislação brasileira para micro-organismos patogênicos. Durante o armazenamento, detectou-se um aumento elevado das contagens de micro-organismos psicrotróficos anaeróbios, atingindo valores da ordem de $10^{7} \mathrm{UFC} \mathrm{g}^{-1}$ amostra já aos 14 dias, porém os consumidores não detectaram alterações sensoriais significativas durante todo o período. Conclui-se que a vida útil de lombo ovino armazenado a $4^{\circ} \mathrm{C}$ é de no mínimo 28 dias.

Palavras-chave: cordeiro, Longissimus dorsi, armazenamento, vida de prateleira, teste de aceitação.

\section{ABSTRACT}

Lamb is commercialized today almost entirely in frozen form, but, considering the current trend to greater demand for convenience products, it becomes more evident the need of availability of chilled cuts of meat in the market. The objective of this study was to evaluate the stability and safety of lamb meat packed under vacuum when stored under refrigeration. The lamb meat samples were packed in high barrier multilayer plastic films and stored under refrigeration $\left(4 \pm 1^{\circ} \mathrm{C}\right)$. The stability was evaluated by means of physical and chemical analysis (lipid oxidation, objective color, $\mathrm{pH}$ value, chemical composition, cooking losses and instrumental texture), microbiological analysis (total count of psychrotrophic anaerobic, coliform count at $45^{\circ} \mathrm{C}$, coagulase-positive staphylococci and the presence of Salmonella) and sensory analysis (color, flavor and overall appearance). The vacuum packed lamb meat remained stable as to most physical and chemical indexes and microbiological indexes showed good stability throughout the 28 days of storage according to Brazilian legislation standards to pathogenic microorganisms. In relation to the stability evaluation of chilled sheep loin, it was detected a high increase of counts for psychrotrophic anaerobic microorganisms, reaching around $10^{7} \mathrm{CFU} \mathrm{g}^{-1}$ sample at 14 days storage, but the pannelists did not detect significant sensory changes during all the period. It is concluded that the shelf life of sheep loin stored at $4^{\circ} \mathrm{C}$, is at least, 28 days.

Key words: lamb, Longissimus dorsi, storage, shelf life, acceptance test.

\section{INTRODUÇÃO}

Mundialmente, a carne ovina representa aproximadamente apenas $7 \%$ de toda a carne produzida,

IPrograma de Pós-graduação em Ciências da Engenharia de Alimentos, Departamento de Engenharia de Alimentos da Faculdade de Zootecnia e Engenharia de Alimentos da Universidade de São Paulo (FZEA/USP), Av. Duque de Caxias Norte, 225, 13.635-900, Pirassununga, SP, Brasil. E-mail: rafaellapaseto@usp.br. Autor para correspondência.

IIDepartamento de Engenharia de Alimentos, FZEA/USP, Pirassununga, SP, Brasil.

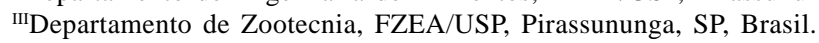

${ }^{\mathrm{IV}}$ Departamento de Ciências Básicas, FZEA/USP, Pirassununga, SP, Brasil. 
sendo que os maiores exportadores são Nova Zelândia e Austrália e os maiores importadores são os países do Sudeste da Ásia e Norte da África. Mesmo possuindo um clima favorável e um pasto altamente produtivo, o Brasil ainda continua sendo um país importador principalmente do Uruguai, Argentina, Chile e Nova Zelândia, e possui um grande potencial para ampliar sua produção interna e para ser um grande produtor e exportador. No Brasil, o consumo per capita anual gira em torno de 0,7kg(MORENO et al., 2008; SANTOS, 2009).

Apesar do consumo ainda pouco expressivo no Brasil, verifica-se que, atualmente, a carne de ovinos vem sendo cada vez mais procurada por um mercado consumidor que paulatinamente vem se tornando cada vez mais exigente em relação à qualidade. Nesse contexto, os frigoríficos devem investir na oferta de cortes e produtos cárneos diferenciados para atender a essa crescente demanda (SANTOS, 2009).

Mesmo com um progressivo aumento do consumo, a carne ovina é comercializada, na maioria dos casos, principalmente no Estado de São Paulo, na forma congelada. Com a tendência atual de maior procura por produtos de conveniência, vislumbra-se a necessidade do mercado começar a ofertar a carne ovina na forma de cortes resfriados. Porém, além de um período com vida útil muito menor, se comparada com a carne congelada, que pode permanecer estocada por vários meses, o produto refrigerado é susceptível a alterações, que comprometem suas características físicas, químicas e microbiológicas.

Diante do exposto, o objetivo deste trabalho foi avaliar a estabilidade física e química, microbiológica e sensorial da carne ovina quando armazenada sob refrigeração $\left(4 \pm 1^{\circ} \mathrm{C}\right)$ por 28 dias, visando a possibilitar uma comercialização segura e conveniente ao consumidor final, que requer praticidade.

\section{MATERIAL E MÉTODOS}

As amostras de lombos desossados de carne ovina foram obtidas de cortes realizados entre a 1e e 6 vértebras lombares (Longissimus lumborum). As amostras foram embaladas a vácuo em sacos de filmes plásticos termoencolhíveis de alta barreira e armazenadas durante um período de 28 dias em temperatura de $4 \pm 1^{\circ} \mathrm{C}$. O experimento foi realizado em três repetições, sendo as análises realizadas em intervalos de estocagem de 7 dias $(0,7,14,21$ e 28 dias). Os resultados obtidos foram avaliados por análise de variância (ANOVA) e as médias foram comparadas através do teste de Tukey, em nível de 5\% de significância. As respostas experimentais obtidas foram submetidas a ajustes de modelos de regressão para verificar as variações ocorridas ao longo do período de estocagem.

\section{Análises microbiológicas}

Foram realizadas as análises de contagem total de micro-organismos psicrotróficos anaeróbios de acordo com a metodologia descrita por JOHNSTON \& TOMPKIN (1992) e os outros micro-organismos segundo AOAC (2007). A presença de Salmonella foi determinada por método rápido a partir de préenriquecimento (AOAC 2003.09), estafilococos coagulase positiva pelo método AOAC 2003.11 e coliformes termotolerantes a $45^{\circ} \mathrm{C}$, através de AOAC 998.08 .

\section{Análises físicas e químicas}

Para a análise de cor objetiva, utilizou-se um colorímetro portátil (Modelo MiniScan XE, Marca HunterLab), através da escala $L^{*}$, a* e b* do sistema de avaliação CIELab, utilizando-se o iluminante $\mathrm{D}_{65}$, ângulo de observação de $10^{\circ}$ e abertura de célula com $30 \mathrm{~mm}$, para a leitura em seis pontos distintos, após 30 minutos de exposição da superfície das amostras ao ar atmosférico.

As medições de $\mathrm{pH}$ foram realizadas através de pHmetro (Modelo HI 99163, Marca HANNA) com eletrodo combinado para leitura em triplicata com perfuração. Estas mesmas amostras foram submetidas à avaliação da extensão da oxidação lipídica através do teste de TBARS (substâncias reativas ao ácido 2tiobarbitúrico), seguindo-se metodologia de VYNCKE (1970), sendo os resultados expressos em mg de malonaldeído $\mathrm{kg}^{-1}$ de amostra.

Para a análise da composição centesimal, a metodologia oficial da AOAC (2007) foi utilizada para determinação de umidade (950.46), resíduo mineral fixo ou cinzas (920.153) e proteínas (981.10). O teor de lipídeos foi determinado pelo método de BLIGH \& DYER(1959).

Quanto à avaliação de perda de água por cocção (PAC), as amostras foram assadas em um forno elétrico, à temperatura de $180^{\circ} \mathrm{C}$, até atingir $72^{\circ} \mathrm{C}$ internamente (centro geométrico). A PAC foi determinada através da equação: PAC $=[($ Peso inicial - peso final)/peso inicial)] x 100, sendo os resultados expressos em porcentagem. Após cocção, as amostras foram cortadas individualmente, e paralelamente às fibras musculares, em 10 paralelepípedos com dimensões de $2 \times 1 \times 1 \mathrm{~cm}$, sendo estes submetidos a texturômetro Warner Bratzler para a determinação da força de cisalhamento, expressa em kg. 
Análise sensorial

Inicialmente, efetuou-se o recrutamento de provadores não treinados, sendo selecionados aqueles que declararam gostar de carne ovina. Para a avaliação da estabilidade sensorial durante a estocagem, as amostras eram coletadas e congeladas a cada sete dias. Ao final do período de armazenamento (28 dias), as carnes eram descongeladas e avaliadas na forma in natura. Para tanto, foi empregado o método afetivo de aceitação com escala hedônica de nove pontos, sendo avaliados apenas atributos visuais (cor e aparência geral) e aroma, de acordo com metodologia descrita por MEILGAARD et al. (1991).

Para os ensaios sensoriais, as carnes embaladas foram colocadas em caixas de isopor com tampa, para avaliação da aparência geral e a cor da amostra, simulando condições de compra. Em seguida, o provador era solicitado a avaliar odor da amostra em embalagem previamente aberta, simulando a abertura da embalagem no momento do preparo da carne.

\section{RESULTADOS E DISCUSSÃO}

Quanto à composição centesimal, as amostras apresentaram uma porcentagem e desvio padrão de 76,15 $\pm 0,08,1,08 \pm 0,00,1,99 \pm 0,02$ e 20,9 $\pm 0,2$, respectivamente, para umidade, cinzas, lipídeos e proteínas. Estes valores encontrados foram compatíveis com diversos relatos da literatura científica (MADRUGA et al., 2006; JARDIM et al., 2007), sendo o valor percentual de umidade encontrado pelos autores entre 74 e $76 \%$ e de cinzas entre 0,98 e 1,2\%, o de proteína variou entre 20 e $23 \%$ e lipídeos entre 2 e $4 \%$.

Estabilidade microbiológica

Durante todo o período avaliado, não foram detectadas contagens de estafilococos coagulase positiva (<10UFC $\mathrm{g}^{-1}$ de amostra) nem presença de Salmonella. Com relação aos coliformes termotolerantes, somente foram detectadas contagens esporádicas de $3,5 \times 10^{1}$ e 2,0x10 ${ }^{1} \mathrm{UFC} \mathrm{g}^{-1}$ amostra nos intervalos 7 e 14 dias, respectivamente, refletindo uma possível contaminação durante a manipulação das amostras. Sendo assim, a carne de lombo de cordeiros manteve-se dentro dos limites aceitáveis estabelecidos pela legislação brasileira através da Resolução RDC n.12 de 02 de janeiro de 2001 (BRASIL, 2001), cujas contagens previstas para carnes embaladas a vácuo, não maturadas, são $10^{4}$ para coliformes termotolerantes, $3 \times 10^{3}$ para estafilococos coagulase positiva e ausência de Salmonella em 25g de amostra.

Com relação à contagem de psicrotróficos anaeróbios, observou-se variação significativa $(\mathrm{P}<0,5)$ ao longo do período de estocagem avaliado. Este comportamento pôde ser explicado por um modelo quadrático de regressão $(\mathrm{P}<0,05)$, verificando-se uma multiplicação acentuada até 14 dias (de $10^{1}$ a $10^{7} \mathrm{UFC} \mathrm{g}^{-1}$ amostra), seguida de uma estabilização e ligeira queda nas contagens ao final do experimento (Figura 1). As contagens de 14 a 28 dias de armazenamento foram bastante elevadas (acima de $10^{7} \mathrm{UFC} \mathrm{g}^{-1}$ amostra), como também detectado por NISHI (2008), que, ao estudar a estabilidade de carne bovina embalada a vácuo em diferentes temperaturas $\left(0,2,4,7\right.$ e $\left.10^{\circ} \mathrm{C}\right)$, verificou que aos 14 dias em temperatura de $4^{\circ} \mathrm{C}$, já havia uma contagem de $10^{7} \mathrm{UFC} \mathrm{g}^{-1}$ amostra.

Segundo ROÇA \& SERRANO (1995) e LEITÃO (2003), o início da deterioração pode ser detectado através da descoloração superficial, quando a contagem microbiana atinge cerca de $10^{6} \mathrm{UFC} \mathrm{cm}^{-2}$, sendo que as alterações em relação ao odor podem ser evidenciadas a partir de valores de $10^{7} \mathrm{UFC} \mathrm{cm}^{-2}$; de

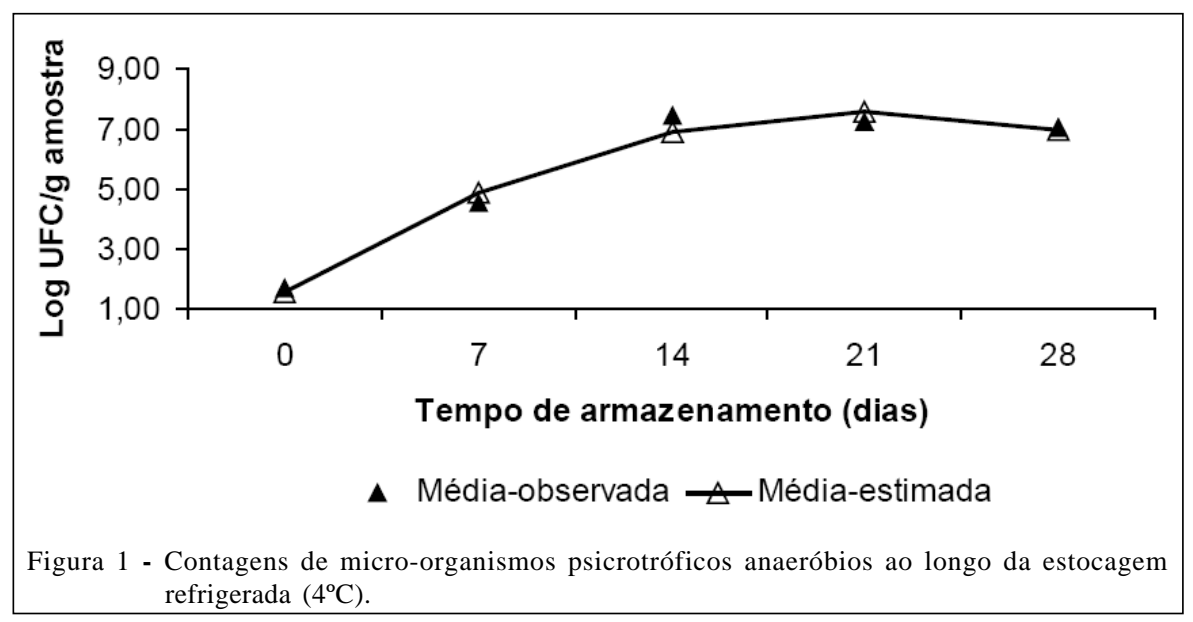

Ciência Rural, v.42, n.4, abr, 2012. 
sabor, $10^{8} \mathrm{UFC} \mathrm{cm}^{-2}$ e a limosidade superficial pode ser observada ao se ter uma população microbiana de $10^{9} \mathrm{UFC} \mathrm{cm}^{-2}$ de amostra. Sendo assim, pode-se considerar que a carne ovina armazenada neste experimento em condições normais de comercialização $\left(4^{\circ} \mathrm{C}\right)$, apesar de não apresentar riscos à saúde do consumidor, mesmo após 28 dias, poderia apresentar sinais de deterioração a partir de 14 dias de armazenamento.

\section{Estabilidade física e química}

De acordo com os resultados encontrados, houve um aumento $(\mathrm{P}<0,05)$ linear na perda de água por cocção (PAC) ao longo da estocagem, passando de $18,24 \%$ no primeiro dia para $28,25 \%$ após 28 dias de armazenamento refrigerado, provavelmente devido à atividade proteolítica causada tanto pelas endopeptidases da própria carne quanto por enzimas produzidas pelos micro-organismos, já que estes atingiram contagens bastante elevadas $\left(10^{7} \mathrm{UFC} \mathrm{g}^{-1}\right.$ amostra). Esta proteólise promove uma baixa retenção de água, levando à progressiva perda de peso durante o período de estocagem (NISHI, 2008). O valor médio de PAC $(24,33 \pm 4,75 \%)$ apresentou-se próximo ao encontrado para músculo Longissimus dorsi de cordeiros em estudos realizados por CANEQUE et al. (2004), que obtiveram uma porcentagem de 26,04\% após estocagem de 48 horas a $4^{\circ} \mathrm{C}$.

Em relação à maciez, o lombo de cordeiro não apresentou diferença significativa $(\mathrm{P}>0,05)$ ao longo do tempo de estocagem, apresentando valores médios de 3,23ะ0,66kg. PETHICK et al. (2005), avaliando a força de cisalhamento dos músculos Longissimus lumborum e semimembranosus de cordeiros de 8,5 meses de idade, armazenados durante 5 dias entre 1 a $3^{\circ} \mathrm{C}$, observaram uma média de 2,45 e $4,65 \mathrm{~kg}$, respectivamente, que são valores próximos à média obtida no presente estudo. O aumento da maciez esperado devido à maturação da carne durante a estocagem refrigerada pode não ter sido detectado nas análises de força de cisalhamento, justamente devido ao aumento na PAC, pois carnes com menor capacidade de retenção de água tendem a ser mais duras após o cozimento.

A oxidação lipídica do lombo ovino durante armazenamento refrigerado, tal qual avaliada pelo índice de TBARS (mg de malonaldeído $\mathrm{kg}^{-1}$ de amostra), apresentou um comportamento de aumento linear $(\mathrm{P}<0,05)$ ao longo do período avaliado. Apesar do aumento verificado, os valores de TBARS observados ficaram sempre abaixo de $0,5 \mathrm{mg}$ de malonaldeído $\mathrm{kg}^{-1}$ de amostra e podem ser considerados aceitáveis. De acordo com SINCLAIR (2007), a carne de ovinos é rica em ácidos graxos saturados e possui um baixo teor de poliinsaturados, o que, segundo ELLIS \& BERTOL (2001), a torna menos propensa à ocorrência da oxidação lipídica. Vale ainda ressaltar o baixo teor de lipídeos (aproximadamente 2\%) presente nas amostras avaliadas. Valores de TBARS até $1,59 \mathrm{mg}$ de malonaldeído $\mathrm{kg}^{-1}$ de amostra são considerados baixos para serem percebidos por análise sensorial e não causam problemas para a saúde do ser humano (TERRA et al., 2006). PETRON et al. (2007), estudando o efeito de pastagens na estabilidade oxidativa de músculo Longissimus thoracis et lumborum de cordeiros envoltos por filme de PVC permeável ao $\mathrm{O}_{2}$ sob refrigeração a $4^{\circ} \mathrm{C}$, após 4 e 8 dias post-mortem, também observaram, assim como no presente estudo, um aumento dos valores durante o armazenamento, sendo que estes não ultrapassaram $0,7 \mathrm{mg}$ de malonaldeído $\mathrm{kg}^{-1}$ de amostra. Da mesma forma, SANTÉ-LHOUTELLIER et al. (2008), analisando o efeito da dieta sobre a estabilidade de músculo Longissimus dorsi de cordeiros, após armazenamento por um período de 7 dias a $4^{\circ} \mathrm{C}$, verificaram a existência de um aumento da oxidação lipídica com o tempo de estocagem em amostras provenientes de animais submetidos à dieta a base de concentrado.

Em relação aos valores de $\mathrm{pH}$ ao longo do tempo de estocagem, não se verificou uma variação significativa $(\mathrm{P}>0,05)$. Durante o período de armazenamento, o valor médio do $\mathrm{pH}$ foi de $5,43 \pm 0,22$, permanecendo dentro da faixa de 5,31 e 5,59, que corresponde aos valores também encontrados por JUAREZ et al. (2008) em amostras de carnes da mesma espécie.

Para a análise de cor objetiva, os valores de $L^{*}, a *$ e b* não variaram significativamente $(\mathrm{P}>0,05)$ durante a estocagem, apresentando médias de $36,52 \pm 2,66,6,96 \pm 1,02$ e 10,46 $\pm 1,43$, respectivamente. Sendo assim, pode-se afirmar que o sistema de embalagem a vácuo protegeu de maneira eficaz os pigmentos da carne contra a ocorrência da oxidação, durante todo o período analisado. Similarmente, estudos realizados por STROHECKER et al. (1997) com músculos Longissimus lumborum de cordeiros, para a determinação dos efeitos da suplementação com e sem vitamina E sobre a estabilidade da cor, demonstraram que esta característica não foi afetada durante os dias 0, 3, 6, 9 e 12 de armazenamento a $4^{\circ} \mathrm{C}$, em ambos os tratamentos.

\section{Estabilidade sensorial}

Não se verificou variação significativa $(\mathrm{P}>0,05)$ na aceitação dos três atributos avaliados (cor, 
aparência e aroma) ao longo dos 28 dias de armazenamento, sendo que os valores para cor permaneceram entre 5,7 e 6,1; para aparência geral, 5,7 a 6,2; e aroma, 6,0 a 6,7, demonstrando que consumidores não treinados não foram capazes de perceber possíveis alterações nestes atributos ao longo do tempo.

Quanto ao atributo cor, a avaliação sensorial afetiva demonstrou uma boa correlação com as medidas objetivas (parâmetros $L^{*}$, a* e b*), já que estas também não variaram $(\mathrm{P}>0,05)$ ao longo do tempo de armazenamento. Por outro lado, em relação aos atributos aparência e aroma, os consumidores não detectaram alterações, mesmo considerando o aumento das contagens de micro-organismos psicrotróficos anaeróbios, que, partindo de $10^{1}$ no início do experimento, apresentaram-se, ao final do período de estocagem, com uma população de $10^{7} \mathrm{UFC} \mathrm{g}^{-1}$ amostra, fator este que poderia ter contribuído para a redução da aceitação destes atributos.

Em geral, os valores médios atribuídos pelos provadores ficaram próximos de 6 ("gostei ligeiramente”) em todos os intervalos avaliados. Levando-se em consideração o valor de cinco na escala sensorial ("nem gostei/nem desgostei”) como o limite de aceitação para o lombo de cordeiro embalado a vácuo, pode-se considerar que este permaneceu aceitável mesmo após 28 dias de armazenamento refrigerado. Similarmente a este estudo, VERGARA et al. (2002) também verificaram que, em amostras de carne de cordeiro embaladas a vácuo e armazenadas sob refrigeração a $2^{\circ} \mathrm{C}$, a cor e o aroma se mantiveram aceitáveis durante todo o período experimental (28 dias).

\section{CONCLUSÃO}

A carne ovina embalada a vácuo apresentouse estável com relação à maioria dos índices físicos e químicos e aos atributos sensoriais avaliados e dentro dos padrões estabelecidos pela legislação brasileira para micro-organismos patogênicos durante os 28 dias de estocagem refrigerada. No entanto, considerando as contagens de micro-organismos deterioradores acima de $10^{7} \mathrm{UFC} \mathrm{g}^{-1}$ amostra, é possível concluir que a vida útil do lombo ovino embalado a vácuo e armazenado a $4^{\circ} \mathrm{C}$ é de aproximadamente 14 dias.

\section{AGRADECIMENTOS}

À Fundação de Amparo à Pesquisa do Estado de São Paulo (FAPESP), pelo auxílio financeiro (Programa de Pesquisa em Políticas Públicas - Processo n. ${ }^{\circ}$ 06/51695), à CRYOVAC, pelo fornecimento das embalagens utilizadas neste estudo e à Coordenação de Aperfeiçoamento de Pessoal de Nível Superior (CAPES), pela concessão da bolsa de estudos.

\section{COMITÊ DE ÉTICA E BIOSSEGURANÇA}

Apesar do produto desta pesquisa envolver análise sensorial com participação de seres humanos, apenas atributos visuais e olfativos foram avaliados, sem o consumo da carne. Dessa maneira, os consumidores não foram expostos a quaisquer riscos, portanto, dispensando a submissão do projeto ao comitê de ética em pesquisa com seres humanos.

Da mesma forma, as carnes foram adquiridas diretamente de frigorífico comercial, que atende aos preceitos de ética e bem-estar animal. Como neste projeto não houve manipulação direta dos animais vivos, também não foi submetido ao comitê de ética em pesquisa envolvendo animais.

\section{REFERÊNCIAS}

ASSOCIATION OF OFFICIAL ANALYTICAL CHEMISTRY (AOAC). Official methods of analysis. 18.ed. Gaithersburg: AOAC International, 2007. xxix, 1v. (various pagings).

BRASIL. Leis, decretos, etc. Resolução RDC n.12, 02 de janeiro de 2001 da Agência Nacional de Vigilância Sanitária. Aprova o Regulamento Técnico sobre Padrões Microbiológicos para Alimentos. Diário Oficial, Brasília, 10 jan. 2001, Seção 1, p. $45-47$.

BLIGH, E.G.; DYER, W.J. A rapid method of total lipid extration and purification. Canadian Journal of Biochemistry and Physiology, Ottawa, v.37, n.8, p.911-917, 1959.

CANEQUE, V. et al. Carcass and meat quality of light lambs using principal component analysis. Meat Science, Barking, v.67, n.4, p.595-605, 2004. Disponível em: <http://dx.doi.org/ 10.1016/j.meatsci.2004.01.002>. Acesso em: 20 jul. 2010. doi:10.1016/j.meatsci.2004.01.002.

ELLIS, M.; BERTOL, T.M. Efeitos do peso de abate sobre a qualidade de carne suína e da gordura. In: CONFERÊNCIA INTERNACIONAL VIRTUAL SOBRE QUALIDADE DE CARNE SUÍNA, 2., 2001, Concórdia, SC. Anais... Concórdia: EMBRAPA Suínos e Aves, 2001. 13p. p.236.

JARDIM, R.D. et al. Composição tecidual e química da paleta e da perna em ovinos da raça Corriedale. Revista Brasileira de Agrociência, Pelotas, v.13, n.2, p.231-236, 2007.

JOHNSTON, R.W.; TOMPKIN, R.B. Meat and poultry products. In: VANDERZANT, C.; SPLITTSTOESSER, D.F. Compendium of methods for the microbiological examination of foods. Washington: APHA, 1992. Cap.44, p.821-835.

JUAREZ, M. et al. Southern Spain lamb types discrimination by using visible spectroscopy and basic physicochemical traits. Meat Science, Barking, v.80, n.4, p.1249-1253, 2008. Disponível em: <http://dx.doi.org/10.1016/j.meatsci.2008.05.033>. Acesso em: 15 maio, 2010. doi: 10.1016/j.meatsci.2008.05.033.

LEITÃO, M.F.F. Aspectos microbiológicos das carnes. In: CONTRERAS, C.J. et al. Higiene e sanitização na indústria de carnes e derivados. São Paulo: Varela, 2003. Cap.1, p.1-5.

MADRUGA, M.S. et al. Efeito do genótipo e do sexo sobre a composição química e o perfil de ácidos graxos da carne de cordeiro. Revista Brasileira de Zootecnia, Viçosa, v.35, 
n.4, p.1838-1844, 2006. Disponível em: <http://www.scielo.br/ scielo.php?script=sci_arttext\&pid=S151635982006000600035\&lng= en\&nrm=iso>. Acesso em: 18 jan. 2010. doi: 10.1590/S151635982006000600034

MEILGAARD, M.C. et al. Sensory evaluation techniques. Boca Raton: CRC, 1991. 287p.

MORENO, G.M.B. et al. Características qualitativas da carne ovina. Revista Nacional da Carne, São Paulo, n.381, p.7690, 2008

NISHI, L.M. Efeito da temperatura de estocagem sobre a estabilidade de carne bovina (M. Gluteus medius) embalada a vácuo. 2008. 138f. Dissertação (Mestrado em Tecnologia de Alimentos) - Curso de Pós-graduação em Tecnologia de Alimentos, Universidade Estadual de Campinas, SP.

PETHICK, D.W. et al. Effects of animal age on the eating quality of sheep meat. Australian Journal of Experimental Agriculture, Melbourne, v.45, n.5, p.491-498, 2005. Disponível em: <http://dx.doi.org/10.1071/EA03256>. Acesso em: 25 out. 2010. doi: 10.1071/EA03256.

PETRON, M.J. et al. Effect of grazing pastures of different botanical composition on antioxidant enzyme activities and oxidative stability of lamb meat. Meat Science, Barking, v.75, n.4, p.737-745, 2007. Disponível em: <http://dx.doi.org/ 10.1016/j.meatsci.2006.10.010>. Acesso em: 25 out. 2010 doi:10.1016/j.meatsci.2006.10.010.

ROÇA, R.O.; SERRANO, A.M. Abate de bovinos: alterações microbianas da carcaça. Higiene Alimentar, São Paulo, v.9, p.589-596, 1995

SANTÉ-LHOUTELLIER, V. et al. Assessment of the influence of diet on lamb meat oxidation. Food Chemistry, London, v.109, n.3, p.573-579, 2008. Disponível em: <http://dx.doi.org/ 10.1016/j.foodchem.2007.11.081>. Acesso em: 08 set. 2010. doi:10.1016/j.foodchem.2007.11.081.
SANTOS, J.A. Muito espaço para crescer. A granja do ano, Porto Alegre, n.23, p.76-77, 2009.

SINCLAIR, L.A. Nutritional manipulation of the fatty acid composition of sheep meat: a review. Journal of Agricultural Science, Cambridge, v.145, n.5, p.419-434, 2007. Disponível em: <http://dx.doi.org/10.1017/S0021859607007186>. Acesso em: 13 mar. 2009. doi: 10.1017/S0021859607007186.

STROHECKER, M.G. et al. Vitamin e supplementation effects on color and lipid stability of whole and ground lamb. Journal of Muscle Foods, Trumbull, v.8, n.4, p.413-426, 1997. Disponível em: <http://dx.doi.org/10.1111/j.17454573.1997.tb00729.x>. Acesso em: 22 jan. 2010. doi: 10.1111/j.1745-4573.1997.tb00729.x.

TERRA, N.N. et al. Valores de nitrito e de TBARs durante o processo e armazenamento da paleta suína curada, maturada e fermentada. Ciência Rural, Santa Maria, v.36, n.3, p.965970, 2006. Disponível em: <http://www.scielo.br/ scielo.php ? script=sci_arttext \& pid=S 0103 $84782006000300037 \& \operatorname{lng}=$ en\&nrm=iso>. Acesso em: 26 jun. 2010. doi: 10.1590/S0103-84782006000300037.

VERGARA, H. et al. Evolución de los parámetros de calidad de la carne de cordero de raza manchega conservada en vacío. In: JORNADAS CIENTÍFICAS Y VI JORNADAS INTERNACIONALES DE LA SOCIEDAD ESPAÑOLA DE OVINOTECNIA Y CAPRINOTECNIA (SEOC), 27., 2002, Valência, CA. Atas... São Paulo: Gráficas Marí-Montanyana, 2002. 1143p. p.388-393.

VYNCKE, B.W. Direct determination of the thiobarbituric acid value in trichloracetic acid extracts of fish as a measure of oxidative rancidity. Fette Seifen Anstrichmittel, Leinfelden, v.72, n.12, p.1084-1087, 1970. Disponível em: <http:// dx.doi.org/10.1002/lipi.19700721218>. Acesso em: 26 jun. 2010. doi: 10.1002/lipi.19700721218. 